\title{
Solar Monitoring has a Past and Present: Does it have a Future?
}

\author{
By RICHARD C. WILLSON
} Solar Irradiance Monitoring Group, Earth and Space Sciences Division, Jet Propulsion
Laboratory, California Institute of Technology, Pasadena, CA 91109, USA

Intrinsic variations of total solar irradiance have, after nearly a century of sustained effort, been demonstrated by flight experiments during solar cycles 21 and 22. This accomplishment has been the result of a collaborative effort on behalf of several groups of researchers in both the United States and Europe. The overriding concern at this point of time is whether the integrity of the precision long-term solar total irradiance database, which began at the maximum of solar cycle 21 and continues in the present, will be lost in the late 1990's, prior to the inception of experiments planned for the NASA Earth Observing System beginning in 2002. Experiments are not currently in place to prevent an unbridgeable discontinuity in the precision total solar irradiance database.

\section{Introduction}

The desire to monitor total solar irradiance (TSI), the energy supplied to the Earth by the Sun, has been a goal of mankind for more than a century. Interest in possible variability in this quantity arose from a realization that it is a primary determining factor for the Earth's climate. At any time in the Earth's history, the total solar irradiance, atmospheric chemical composition, and the distribution of oceans and land masses act in combination to determine the radiative balance, and hence the climate, of the biosphere.

In recent years the science of paleoclimatology has made great advances in understanding past climate changes and their causal mechanisms. On the timescale of billions of years, solar luminosity is thought to have increased about $30 \%$ during the evolutionary cycle of the Sun. About a billion years before present, the increase in total solar irradiance combined with changes in the composition of the Earth's atmosphere to provide a climate that could support life (Newkirk 1982). The largest climate changes during the existence of life on Earth were most likely of non-solar origin. Some were caused by volcanic-driven sea floor spreading that moved the continental land masses and changed the topology of the ocean basins. The resulting alterations of ocean circulation, the primary heat exchange mechanism for the Earth's climate, resulted in major climate changes. Others may have been caused by the impact of extra-terrestrial objects such as asteroids or large meteorites. The most recent large-scale climate change began about 65 million years ago and continues into the present.

On timescales between 19 and 100 hundred thousand years, periodic variations in the Earth's orbit, the so-called Milankovich effects, have induced an effective variation of the total solar irradiance of the Earth. The record of resulting climate variations has been inferred from the stratigraphy of benthic formanifera and the ratio of oxygen isotopes $(16 / 18 \mathrm{amu})$ found in cores of sea floor sediment. While this is not an example of intrinsic solar variability and its climatic effect, it is a dramatic example of the climate impact of subtle variations of solar irradiance (Hays et al. 1976).

The magnetic 'activity' cycle of the Sun is known to have varied in periodicity and magnitude. Variations over the past ten thousand years are chronicled by the varying abundances of 'cosmogenic' isotopes, notably carbon 14 in living and fossil organic materials and beryllium 10 in Greenland ice cores. Periodicities near 11, 88, 208 and 2300 
years have been found (Damon \& Sonnet 1991; Beer et al. 1990). During the past millennia, variations of solar activity are correlated with periods of significant climate change. While TSI variations were suspected as the cause of these changes, direct observations linking TSI and solar activity variation was not available until recently (Willson \& Hudson 1988).

\section{Attempts to measure total solar irradiance variability}

Attempts were made to detect TSI variability from ground-based observatories, most notably by the Smithsonian Astrophysical Observatory, over the first half of the 20th century (Abbot \& Fowle 1908; Hoyt 1979). During the following 30 years, experiments with the same objective were conducted using aircraft, high-altitude balloons, sounding rocket and space flight platforms (Fröhlich 1976). All were unable to detect total irradiance variations that were unambiguously solar in origin. Failure of the intra-atmospheric experiments was caused by uncertainties of atmospheric attenuation for the TSI that masked solar variations from even high flying aircraft and balloons. Failure of extra atmospheric experiments on rockets and early satellites was due to limitations in accuracy and precision of early solar monitoring flight instrumentation (Willson 1984).

Two developments following the end of the Smithsonian ground-based program facilitated the modern exploration of solar total irradiance variations: the development of new, more accurate and precise TSI detectors, and the availability of space flight platforms capable of supporting sustained observations.

The first of a new generation of total solar irradiance measurement instrumentation, so-called electrically self-calibrating cavity (ESCC) detectors, was developed at the Jet Propulsion Laboratory (JPL) in the mid 1960's as part of its effort to understand the thermal behavior of interplanetary spacecraft. Several versions of these new "pyrheliometers" were subsequently developed at JPL for different applications: the Thermal Control Flux Monitor (TCFM) for the Mariner 1969 mission (Plamondon 1969), the Practical Cavity Radiometer (PACRAD) for laboratory measurements (Kendall \& Berdahl 1970) and the Active Cavity Radiometer (ACR) for flight observations of TSI on balloon, rocket, shuttle and satellite platforms (Willson 1971, 1984). Similar detectors were developed at other laboratories during the early 1970's: at the Physical Meteorological Observatory at Davos (PMOD - Switzerland) (Brusa \& Fröhlich 1972), the National Bureau of Standards (NBS - USA) (Geist 1972), the Eppley Laboratory (EL - USA) (Hickey \& Karoli 1974) and the Royal Meteorological Institute of Belgium (RMIB) (Crommelynck 1973). An experiment was conducted in 1975 to establish a TSI radiation scale (the World Radiation Reference) embodying the performance of five of these new sensors: three from JPL and one each from the PMOD and RMIB (Brusa \& Fröhlich 1975).

\section{Observations of total solar irradiance variability}

The first implementation of ESCC pyrheliometry in a space flight environment was by the Jet Propulsion Laboratory's TCFM experiments on the Mariner 6 and 7 spacecraft in 1969. A non-ESCC solar detector was included in the Nimbus-6/ERB experiment in 1975. Both were unable to detect intrinsic solar variability due to limitations in instrument accuracy, calibration, precision and sensor degradation (Plamondon 1969).

The first long-term solar monitoring utilizing an ESCC sensor was the Earth Radiation Budget (ERB) experiment on the NOAA Nimbus-7 spacecraft. The ERB solar database, beginning in late 1978 and continuing to early 1993, is the longest currently available (Kyle et al. 1993). Limitations imposed on ERB solar observations by the absence of solar 
pointing on the Nimbus platform sustained a noise level in the ERB results that inhibited recognition of intrinsic solar variability until subsequent detection by JPL's Active Cavity Radiometer Irradiance Monitor I (ACRIM I) experiment on the NASA Solar Maximum Mission (SMM) in 1980 (Willson et al. 1981). The mutually corroborative function of the ACRIM I and ERB results has played an important role in verifying solar variability on solar activity cycle timescales.

A series of shorter-term TSI experiments has been flown on or deployed from the Space Shuttle to provide comparison experiments for satellite monitors. The Spacelab 1 and ATLAS flights between 1983 and 1993 employed two different TSI experiments, as has the shuttle-deployed EURECA platform that operated for 10 months starting in mid 1992 (Willson 1994; Fröhlich 1994; Crommelynck et al. 1994). The shuttle ACRIM experiment has demonstrated a capability of sustaining satellite TSI experiments with less than 300 parts-per-million ( $\mathrm{ppm}$ ) uncertainty, a precision comparable or superior to other methods (including radiometers operating at cryogenic temperatures) except for overlapping, long duration satellite experiments.

\section{Results of total solar irradiance variability observations}

The most significant finding from the precision TSI database thus far is on solar cycle timescales: a direct correlation of luminosity and solar activity (Willson \& Hudson 1988, 1991). With an $0.1 \%$ peak-to-peak amplitude during solar cycle 21 , it agrees in sense with that predicted from the coincidence of the "Little Ice Age" climate anomaly and the "Maunder Minimum" of solar activity during the 16th and 17th centuries.

Solar cycle TSI variation is predicted with varying degrees of success by regression models using the precision TSI database and 'proxies' of solar activity, such as the Zürich sunspot number, the $10.7 \mathrm{~cm}$ microwave flux, He I $1083 \mathrm{~nm}$ flux and the 'core-to-wing ratio' of $\mathrm{Mg}$ II. The use of the $\mathrm{He}$ I model led to the initial realization of the primary role of faculae and the bright network in the solar cycle TSI variation (Foukal \& Lean 1988, 1990; Livingston et al. 1988; Fröhlich \& Pap 1989; Willson \& Hudson 1991).

As useful as these statistical 'proxy' models have been, however, it is not surprising in view of their essentially non-physical nature that significant errors are found in some model predictions. Underestimation of TSI has been a characteristic flaw near the maxima of solar cycles 21 and 22 . Use of models based on proxies with long time series, such as the sunspot area, has tempted some modelers to make tenuous extrapolations into the past, regardless of the highly uncertain character of early proxy observations.

An inverse relationship between sunspot area and total irradiance has been found on the solar rotational timescale (27 days) with deficits in total irradiance of as much as $-0.3 \%$ (Willson et al. 1981; Willson 1982). There is growing evidence that most of the missing flux is balanced by excess facular radiation on the active region timescale (months) with the rest re-distributed through the bright network on the solar cycle timescale.

On the shortest timescales, solar global oscillations of low degree have been detected in the ACRIM I total irradiance data, including pressure modes (timescales of minutes - the so-called 5-minute oscillations or 'p-modes') (Hudson \& Woodard 1983; Woodard \& Hudson 1983) and possible gravity modes (timescales of hours to days) (Fröhlich 1987). Interpretation of the 5-minute oscillation results from the ACRIM I experiment has placed an upper limit on the interior rotation rate of the Sun, and therefore on solar oblateness, providing support for the relativistic interpretation of the precession of the perihelion of Mercury. The p-mode oscillations are concentrated in the convection zone. Therefore the depth within the Sun for which their analysis can provide new physical 
insight is limited. Should gravity mode oscillations be verified in TSI data, however, information on physical processes extending to the solar core would be accessible.

Timescales shorter than a year do not appear to be of direct climatological interest, but contain information on solar variability that provided much new insight into the physics of the Sun. Continuous TSI monitoring, particularly by satellites with a high duty cycle of solar pointing during each orbit, can provide the observations that will facilitate future solar models that can predict TSI variability with sufficient precision to anticipate corresponding climate variations.

\section{Present and planned total solar irradiance monitoring}

The precision TSI database is being sustained at present by two experiments: the NASA ERBS experiment launched in 1984, and the JPL/NASA Upper Atmosphere Research Satellite (UARS)/ACRIM II experiment launched in 1991. The Nimbus-7/ERB experiment, after more than 14 years of operation, ceased acquiring TSI data in early 1993. The next planned satellite TSI experiment is the ESA SOHO/VIRGO (1995 launch), to be followed by three ACRIM experiments in the NASA Earth Observation System CHEM platforms (beginning in 2002). Annual underflights aboard the Space Shuttle by ACRIM and CROM instrumentation will continue at least through 1994 .

\section{Sustaining the total solar irradiance database}

The detection of solar luminosity variability proportional to magnetic activity during solar cycles 21 and 22 provides the impetus to extend the irradiance database with maximum precision. While the $0.1 \%$ peak-to-peak variation during these cycles may be of minor climatological interest, the direct correlation between activity and luminosity implicates TSI variations as the causal mechanism for known frequencies of climate changes. If that is the case there must be other luminosity variabilities with longer periodicities and proportionately larger amplitudes that have significant climatic implications. Subtle trends in the total irradiance of as little as \pm 0.5 percent per century could eventually produce the extreme range of climates known to have existed in the past, from warm periods without permanent ice, to the great ice ages (Eddy 1976).

Accumulation of a century-long database capable of detecting TSI trends of climatological significance, with the continuity required for development of a physically meaningful solar TSI model, will necessarily be composed of the results of many individual solar monitoring experiments. If these experiments last between three and ten years each, the results of from ten to twenty will have to be related with adjacent precisions of 50 to less than $20 \mathrm{ppm}$ to resolve a century of change at the $0.5 \%$ level with a 10:1 signal-to-noise ratio. A careful measurement strategy will be required to sustain the required precision for the TSI database since the absolute uncertainty of current satellite instrumentation is inadequate for this purpose.

The absolute uncertainty (relative to S.I. units) of the current generation of flight TSI instrumentation, which operates at ambient temperatures, is about $1000 \mathrm{ppm}$ in the laboratory and about $2000 \mathrm{ppm}$ in flight experiments. The absolute uncertainty of a new generation of TSI sensors operating near the temperature of liquid Helium approaches $100 \mathrm{ppm}$ in the laboratory (Martin \& Fox 1994). These cryogenic sensors face some significant hurdles in the effort to transform them into space flight experiments, however. They must use much smaller apertures $(0.3 \mathrm{~cm}$ diameter $)$ than their laboratory versions to minimize solar input that would otherwise prevent the space flight qualified stirling cycle 'refrigerators' from maintaining temperatures below $20^{\circ} \mathrm{K}$. Such small apertures 
are difficult to make and measure accurately and they are much more susceptible to contamination than the large-apertured $(0.8 \mathrm{~cm}$ diameter $)$ ambient temperature sensors. A realistic expectation for their eventual in-flight performance would be in the several hundred ppm uncertainty range. Clearly, the absolute uncertainty of neither the ambient or cryogenic temperature TSI sensor technology is adequate to sustain a contiguous longterm database.

A relative precision of less than $50 \mathrm{ppm}$ is achievable for the data of overlapped solar monitors with the current state-of-the-art ambient temperature instrumentation, given a sufficient number of overlapping comparisons (Willson 1994). The principal source of uncertainty for the 'overlap strategy' is degradation of solar monitoring sensors over multi-year missions. The ACRIM experiments have demonstrated that calibration of such degradation is possible at the level of a $50 \mathrm{ppm}$ per decade or less (Willson \& Hudson 1991).

It is clear that the only available monitoring approach capable of supporting the contiguous TSI database is one that relates successive solar monitoring experiments at a precision level defined by the operation of the instrumentation in the space flight environment. The method of choice is the overlapping and comparison of successive solar monitoring experiments: a so-called 'overlap strategy'. Failing that, relating successive TSI monitors using results from a 'third party' experiment that overlaps both would be next best, but with inherent additional uncertainty.

A logical role for cryogenic instrumentation, at least during its developmental stage, is as a shuttle-based underflight experiment. A relatively inexpensive experiment based on the use of expendable cryogens could be used to test the technology and provide periodic calibrations of ambient temperature satellite TSI monitors with enhanced accuracy.

The 'overlap strategy' was to have begun with the SMM/ACRIM I and UARS/ACRIM II experiments. Unfortunately the SMM mission ended in late 1989, two years before UARS could be launched (the result of the Space Shuttle hiatus following the Challenger loss). The relationship between the ACRIM I and ACRIM II experiments has instead been established using a 'third party' overlap strategy using the results of comparisons of both ACRIM I and ACRIM II with the Nimbus-7/ERB experiment. The ratio of ACRIM II to ACRIM I is 1.001759 with a statistical uncertainty smaller than $10 \mathrm{ppm}$, a demonstration of the capability of the 'overlap strategy'.

The Nimbus-7/ERB experiment ceased acquiring data in early 1993 . The outlook for current TSI monitoring experiments by ACRIM II and ERBS must be viewed with a degree of pessimism. ERBS has been in flight nearly a decade, it could fail at any time without surprising anyone. Several problems have already surfaced with the UARS satellite: the batteries are deteriorating faster than anticipated, and the primary solar panel drive system has failed. Neither may last into the SOHO/VIRGO timeframe of 1995 and beyond. SOHO is assumed to be at least a 3-year minimum mission, but its follow-on, EOS/ACRIM experiment, is not scheduled until 2002 in current plans. The probability of a TSI data gap in the mid or late 1990's or early 2000's is high.

Additional solar monitoring experiments are needed between 1994 and 2003 to guarantee continuity of the high-precision TSI database. Failure to implement them may produce an unbridgeable gap in the long-term TSI database at the level of precision required to provide a contiguous, long-term TSI database for climate and solar physics. If a gap in monitoring occurs it will become necessary to start the high precision TSI database over again in 2003, with the relationship between it and the current database unknown with useful precision. 


\section{Recommendations for saving the total solar irradiance database}

A combination of the continuation of one and the addition of a TSI Mission comprised of a series of new, low cost flight experiment activities during the mid-to-late 1990's could provide a high probability of preserving the contiguous TSI database. The first would be the continuation of the annual flights of the shuttle TSI comparison instrumentation beyond its currently planned third and final flight in the ATLAS series of missions in 1994. The ATLAS/ACRIM experiment can sustain a year-to-year precision of better than $300 \mathrm{ppm}$. This is far short of the $20-50 \mathrm{ppm}$ desired, but much superior to the $2000 \mathrm{ppm}$ of ambient TSI experiment accuracies, and comparable to the probable performance of flight versions of the cryogenic TSI experiments.

The first in the series of new flight experiments would have to be launched soon to guarantee overlap with UARS/ACRIM II and SOHO/VIRGO. New small satellite and TSI instrumentation developments make this a potentially feasible, science-effective and cost-effective possibility. Ideally, two TSI satellites should be launched initially. Following comparison, one of the two small satellites would continue monitoring on a full time basis and the other would be saved (except for periodic re-comparisons) as backup for the first. Ground spares would be launched as replacements upon failure of one of the first two satellites, replacing the reserve satellite capability and adding redundancy. Such a TSI mission approach could provide an optimum long-term monitoring modality.

Acknowledgments. This paper was completed by the Jet Propulsion Laboratory, California Institute of Technology, under a contract with the National Aeronautics and Space Administration.

\section{REFERENCES}

Aввот, C.G. \& Fowle, F. I. 1908 Annals of the Astrophysical Observatory of the Smithsonian Institution, $\mathbf{I I}$.

Brusa, R.W. \& Fröhlich, C. 1972 Entwicklung eines neuen absolutradiometers. Technical Note 1., World Radiation Center, Davos, Switzerland.

Brusa, R.W. \& Fröhlich, C. 1975 Scientific discussions IPC IV, World Radiation Center, Davos, Switzerland.

Beer, J., Blinov, A., Bonani, G., Finkel, R.C., Hofmann, H.J., Lehmann, B., Oeschger, H., Sigg, A., Schwander, I., Staffelbach, T., Stauffer, T., Suter, M. \& WOLFLI, W. 1990 Use of ${ }^{10} \mathrm{Be}$ in polar ice to trace the 11 -year cycle of solar activity: Information on cosmic ray history. Nature 347, 164-166.

Crommelynck, D. 1973 Instrumentation theory of absolute radiometry. $P u b . A$ 81, Royal Meteorological Institute of Belgium, Brussels, Belgium.

Crommelynck, D., Domingo, V., Fichot, A. \& Lee, R. 1994 Irradiance observations from the EURECA and ATLAS experiments. In The Sun as a Variable Star: Solar and Stellar Irradiance Variations (ed. J.M. Pap, C. Fröhlich, H.S. Hudson \& S.K. Solanki). Cambridge University Press, in press.

DAmon, P. \& SonNet, C. P. 1991 Solar and terrestrial components of the atmospheric ${ }^{14} \mathrm{C}$ variation spectrum. In The Sun in Time (ed. C. P. Sonnett, M. S. Giampapa \& M. S. Matthews). pp. 360-388. Univ. of Arizona Press, Tucson, AZ, USA.

EDDY, J.A. 1977 Historical evidence for the existence of the solar cycle. In Solar Output and Its Variation (ed. O.R. White). pp. 51-71, Univ. of Colorado Press, Boulder, CO, USA.

FRöHLICH, C. 1977 Contemporary measures of the solar constant. In Solar Output and Its Variation (ed. O.R. White). pp. 93-110. Univ. of Colorado Press, Boulder, CO, USA.

FRöHLICH, C. 1987 Solar oscillations and helioseismology from ACRIM/SMM irradiance data. In New and Exotic Phenomena (ed. O. Fackler \& J. T. T. Van). pp. 395-402. Gif-sur-Yvette: Editions Frontieres. 
Fröhlich, C. \& PAP, J. M. 1989 Multi-spectral analysis of total solar irradiance variations. Astron. Astrophysics. 220, 272-280.

FröHLICH, C. 1994 Reviews of space observations of total solar irradiance. In The Sun as a Variable Star: Solar and Stellar Irradiance Variations, (ed. J.M. Pap, C. Fröhlich, H.S. Hudson \& S.K. Solanki). Cambridge Univ. Press, in press.

GeIST, J. 1972 Fundamental principles of absolute radiometry and the philosophy of this NBS program. Tech. Note 591-1, USNBS, Gaithersburg, MD.

HAYS, J.D., IMBrie, J. \& ShackLEToN, N. J. 1976 Variations in the Earth orbit: pacemaker of the ice ages. Science 194, 1121-1132.

HICKEY, J. R. \& KAROLI, A. R. 1974 Radiometric calibrations for the Earth Radiation Budget experiment. Appl. Opt. 13, 523-533.

Hoyt, D.V. 1979 The Smithsonian Astrophysical Observatory solar constant program. Rev. of Geophysics and Space Physics 17, 427-458.

HUdSON, H.S. \& WOODARD, M. F. 1983 Solar surface granulation and variations of total solar irradiance. Bull. Amer. Astron. Soc. 15, 715.

Kendall, J. M. \& Berdahl, M. 1970 Two blackbody radiometers of high accuracy. J. Appl. Opt. 9, 1082-1091.

Kyle, H. L., Hoyt, D. V., Hickey, J. R., Maschoff, R. H. \& Vallette, B. J. 1993 Nimbus-7 Earth Radiation Budget calibration history-Part I: the solar channels. NASA Reference Publication 1316, Goddard Space Flight Center, Greenbelt, MD.

Livingston, W., Wallace, L. \& White, O. R. 1988 Spectrum line intensity as a surrogate for solar irradiance variation. Science 240, 1765-1767.

Martin, J. E. \& Fox, N. P. 1994 Cryogenic absolute radiometer, CSAR. Solar Physics, in press.

NEwKIRK, G. A. 1982 The nature of solar variability. In Panel on Solar Variability, Weather and Climate, Studies in Geophysics pp. 33-47. National Academy Press, Washington, D.C., USA.

Plamondon, J.A. 1969 TCFM solar observations on Mariner 69. JPL Space Programs Summary 3, pp. 162. Jet Propulsion Laboratory, Pasadena, CA, USA.

WILLSON, R.C. 1971 The active cavity radiometric scale, the international pyrheliometric scale and the solar constant. J. Geophys. Res. 74, 4325.

WILLSON, R.C. 1982 Solar irradiance variations and solar activity. J. Geophys. Res. 86 43194326.

Willson, R.C. 1984 Measurements of solar total irradiance and its variability. Space Science Reviews 38, 203-242.

WILLSON, R.C. 1994 Irradiance observations from the SMM, UARS and ATLAS ACRIM experiments. In The Sun as a Variable Star: Solar and Stellar Irradiance Variations (ed. J. M. Pap, C. Fröhlich, H. S. Hudson \& S. K. Solanki). Cambridge University Press, in press.

Willson, R.C., Gulkis, S., Janssen, M., Hudson, H. S. \& Chapman, G. A. 1981 Observations of solar irradiance variability. Science 211, 700-702.

Willson, R.C., Hudson, H. S., Fröhlich, C. \& BrusA, R. 1986 Long-term downward trend in total solar irradiance. Science 234, 1114-1117.

Willson, R.C. \& Hudson, H. S. 1988 Solar luminosity variations in solar cycle 21. Nature 332, 810-812.

WiLlson, R.C. \& HuDson, H. S. 1991 The Sun's luminosity over a complete solar cycle. Nature 351, 42-44.

Woodard, M.F. \& Hudson, H. S. 1983 Frequencies, amplitudes and linewidths of solar oscillations from solar total irradiance observations. Nature 318, 449-450. 